\title{
The Design of the Infrared Remote Control Switch
}

\author{
Junli Wang \\ School of Mechanical and Electrical Engineering, Zhengzhou University of Industrial Technology, \\ Zhengzhou 451150, Henan, China \\ E-mail: wangjunli_3690@163.com
}

Keywords: Infrared remote, modulation, demodulation, NE555.

\begin{abstract}
The progress of human civilization and the development of science and technology have brought to us a lot of new products, and also the unlimited convenience to the life. Infrared remote control switch which have become common to us is the most extensive application of a remote control and communication means in our daily lives, such as the mobile infrared information transmission, the remote control of color TV, air conditioning, DVD, fans and other electrical appliances. It is with small size, low power consumption, strong function, low cost and is easy to control and use. Due to this, it bring to the society unlimited business chance. This paper presents a kind of infrared remote control switch design, using infrared remote control transmitter to launch control signals and the reception circuit to receive control signal through which the infrared information realize the corresponding control after amplification, and frequency demodulation. It has a simple circuit and understandable principles, and it is easy for electronic enthusiasts to make, and use in life.
\end{abstract}

\section{Introduction}

With the progress of society and the improvement of living standards, people's demand for material life is higher and higher [1]. In recent years, infrared remote control technology is becoming more and more widely used in household electrical appliances remote control, modern industrial remote control system, modern industrial and agricultural production, home security, automation system, scientific research, national defense industry control and people's daily life. Infrared remote control is not only safe and reliable, but also can effectively isolate electromagnetic interference in industrial environment such as high pressure, radiation, toxic gas and dust [2-3]. The central control components of the infrared remote control device have gradually developed from the early discrete components and integrated circuits to the present single chip microcomputer, and the degree of intelligence is greatly improved. According to the different objects, different remote control modes can be used to save resources and reduce costs. The infrared remote control range is usually a few meters to dozens of meters, increasing the launching power of the transmitting end, and can be used for long distance control to realize long distance infrared remote control. The design of a single way infrared transmitting and receiving system is introduced. The NE555 timer and the phase locked loop integrated chip NE567 are used as the main components [4]. It is suitable for the short distance environment with the low interference and the remote control device [5]. The device is cheap, easy to maintain and reliable, and is a practical electronic device. At the same time, 
it brings a lot of convenience to people's work and life, and has good popularization and application value and economic benefit.

\section{Circuit Design}

\subsection{Design of Infrared Remote Control Emission Circuit}

Fig.1 is a schematic diagram of the infrared remote control transmitting circuit, mainly composed of NE555 timer, key switch, capacitor, resistor, power supply and infrared LED. The core of the transmitting circuit is the NE555 timing chip, which is composed of external resistors and capacitors. When the key switch on the right is pressed, the power supply is switched on, the capacitor C3 is charged and VC3 rises. When VC3 rises to 2/3Vcc, the trigger is reset and the NE555 internal discharge BJT T is connected. At this point, the foot is low, and the capacitance C3 is discharged through R3 and variable resistance R2 and T discharge.

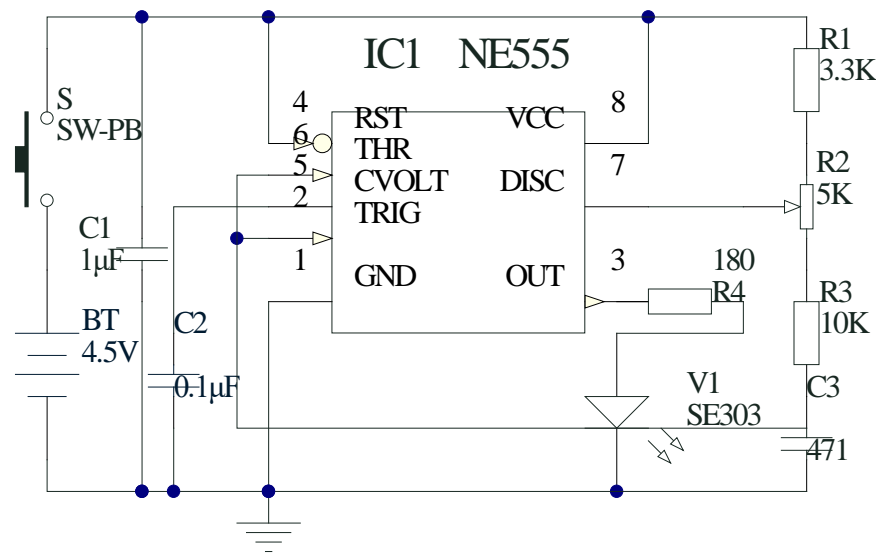

Fig. 1 The infrared remote control transmitting circuit

\subsection{Design of Infrared Remote Control Receiving Circuit}

Fig.2 is the schematic diagram of infrared remote control and receiving circuit, mainly composed of NE567 phase locked loop integrated chip, D trigger, relay, capacitance, resistance and infrared receiving diode.

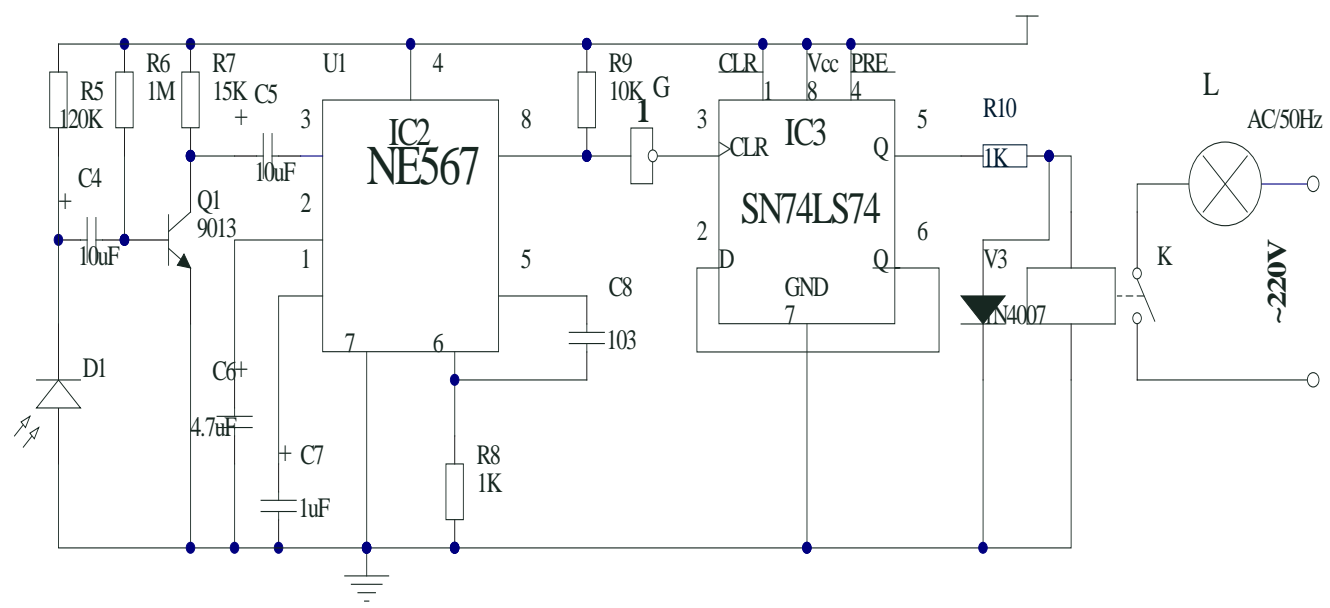

Fig. 2 Infrared remote control and receiving circuit 
The receiving circuit is based on PLL integrated chip NE567, which forms a discriminator circuit. The pulse signal induced by the infrared receiving diode is coupled to the base of the three stage tube Q1 through the capacitance C4. The amplification circuit composed of Q1 amplifies the induction signal for about 100 times and sends it to the NE567's foot, and the frequency discrimination is completed by NE567. When the received signal is within the capture bandwidth of NE567, the pin outputs low level; otherwise, the pin maintains high level. By adjusting the C8 and R8, the capture center frequency of NE567 can be determined and the capture bandwidth of the C6 is changed. The larger the capacitance is, the narrower the bandwidth is captured, and it is particularly important to note that the capacity of the C7 is less than half of the C6. At the same time, the bistable trigger used here is the positive jump edge trigger. At ordinary times, the foot is high level, and the low level of the door is output. When the signal is received, the foot output low level, and when the negative pulse is produced, the no gate output rises along the steep positive pulse, the bistable triggers circuit to change the state, thus controlling the relay and disconnection, and the lighting and extinction of the electric lamp.

\subsection{Power Circuit Design}

Fig. 3 is a schematic diagram of the power supply circuit of the infrared receiving circuit, which is mainly composed of a MC78L05 three terminal voltage regulator, a transformer, a rectifier diode and a capacitor. After the power circuit is connected with $220 \mathrm{~V} \mathrm{AC}$, the transformer is converted into 9V AC power, then the rectifier diode is converted to 9V DC, and then the 5V DC is provided by the capacitor filter and the MC78L05 three voltage regulator.

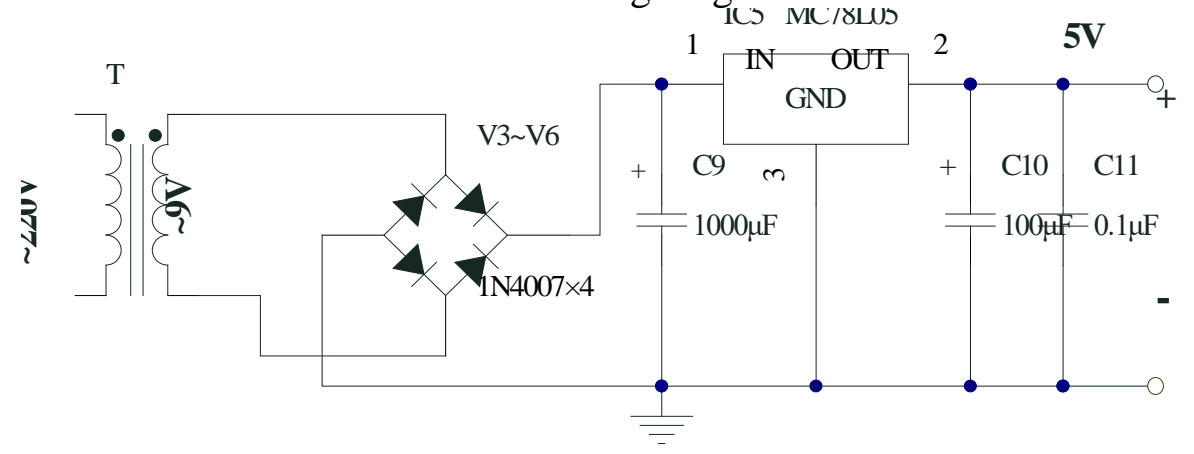

Fig. 3 Power supply circuit

\section{Conclusion}

There is no denying that the age of machinery is over. The electronic age has arrived. Infrared remote control is an important measure of modern household electrical appliances remote control. It is a hot spot of rapid development in recent years. Its rapid development will inevitably bring great economic and social benefits. The infrared remote control system designed in this paper has the advantages of low cost, convenient operation, simple circuit, environmental protection, small volume, light weight and so on. By receiving the control signal of the transmitter, the corresponding control is realized in the receiving part. It has been verified in the experiment. However, this design method cannot be regarded as the most advanced in economic benefit and circuit structure. It is limited by infrared orientation and transmission distance, and is only suitable for short distance ordinary environment remote control. In many aspects, it needs to be improved and improved. 


\section{References}

[1] Konaté C, Kosonen A, Ahola J, et al. Power Line Communication in Motor Cables of Inverter-Fed Electric Drives [J]. IEEE Transactions on Power Delivery, 2010,25(1):125-131.

[2] Junli Wang. Design of an infrared remote control energy-saving switch[A]. Proceedings of 2016 4th International Conference on Advanced Materials and Information Technology Processing (AMITP 2016). 2016,4:213-216.

[3] Howie Huang, Weihua Zhu. Wireless remote control switch[J]. Electrical Technology Magazine,2002 (07): 44-46.

[4] Gengming He. Application of infrared remote control switch technology in intelligent residence [J]. Intelligent Building \& City Information, 2006 (05):124-125.

[5] Jianping Zhang.1 road wireless remote control switch production [J]. Practical Electronics. 2009 (10): $18-20+1$. 